$\xi=-1$

\title{
Conceptual Design of Dosing System for Liquids on the Basis of a Robotic Injection Installation Using Interchangeable Capillaries
}

\author{
Moroz V.V. ${ }^{1}$, Kazachkova O.A. ${ }^{2}$, Baulina L.V. ${ }^{3}$ \\ ${ }^{I}$ Rapid Prototyping Center, Dubna State University,Dubna, Russia, moroz@uni-dubna.ru \\ ${ }^{2}$ MIREA - Russian Technological University, Moscow, Russia \\ ${ }^{3}$ Director of Small Innovative Instrument-Making organization "MULTIAGENT", Dubna,, Russia
}

\section{Introduction}

In laboratory and production conditions, working with liquids of various categories, including dangerous biological fluids and toxic liquid substances, is associated with a number of issues relating to the intake and discharge of a given volume of fluid, for example transfer of fluid to other containers. Given the nature of the fluids with which laboratorians work, it is important to prevent the contamination of liquid samples, disposal of discharged liquid and device elements with surfaces which are in contact with it, as well as limiting the negative impact of hazardous liquids on the operator.

A patent research revealed the presence of solutions related to micro-dosing of liquids [ 1-3 ], which ensure the accuracy of dosing, but do not involve taking a sample of liquid from external (device-independent) tanks; in other words, working only on discharge of liquid [ 1 ], or aiming at multiple intake and discharge of liquid, but without ensuring the sterility of the surfaces in contact with the liquid [ 2 ]. It proposes to use expensive contacting elements in the devices [3 ]. Also, the created solutions do not satisfy the requirements for the robotic system [4].

\section{Creating a New Technical Device}

In this research a technical device is proposed [5] which is a robotic injection unit using replaceable capillaries, the principle of action of which is aimed at solving the following tasks:

1) Improving the accuracy of micro-dosing of liquids in a wide range of costs: previously used for this purpose, needles and pipettes allowed to collect liquid with a volume limited by their own volumes;

2) Preventing contamination of fluid samples;

3) Prevention of possible negative impact of hazardous liquids on personnel, due to the limited contact of operators with hazardous liquids, including the automation of cutting a used capillary and its disposal;

4) Improving the accuracy of dosing, including lessening the influence of the human factor when working with the installation [6,7].

The assigned tasks determine the purpose of creating such a device, which is to increase productivity, reduce cost of consumables, diminish additional operations, and the time spent on research. The process of developing this installation involves development and testing of individual units of system that are responsible for fulfilling the requirements formulated in the patent [ 5 ]. Thus, it is required to design a device which can be used autonomously, working on a laboratory bench, which is also part of an immunochromatographic analysis [8,9] complex to solve the automation problem and, accordingly maximizes exclusion of operator actions [10]. These device requirements are needed for development [11] and determine the following characteristics:

2) Easily portable,

3) Stable on a horizontal surface,

4) It should be possible to fix the unit in the complex of immunochromatographic analysis and movement of the capillary.

\section{Device Development Process}

Design process of a device is an iterative process [12] which includes:

1) Definition of data input due to the requirements of device,

2) Development of the concept of device as a result of research, and analysis of technical solutions used for these purposes,

3) Three-dimensional virtual design,

4) Prototyping,

5) Approbation

6) Identification of new requirements

7) Iteration.

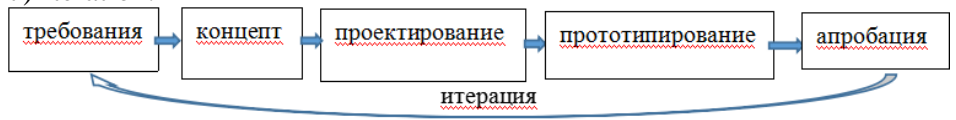

Fig. 1. Design development process

The process of developing concept itself can also be viewed as an iterative [13] course of action, since when conceptual solution for individual units is chosen, it is necessary to revise the design of the plant as a whole each time, and taking into account structural nuances of the individual units and the principles of their interaction [14].

\subsection{Base Blocks of Robotized Injection Installation with Use of Replaceable Capillaries}

Fundamental units of a robotic injection installation using interchangeable capillaries, based on a capillary control system, 
are schematically shown in Figure 2, including a positioning uni (fixing and moving the capillary tip), a used capillary section trimming unit, a sample set block, a microdischer and a sample feed rotor, dosing unit, microbatcher and cassette positioning unit, autonomous operation of the installation providing unit and / or the possibility of using it as part of a complex immunoassay analysis for the automation solution.

\begin{tabular}{|c|c|c|}
\hline BroK HaбOpa tpoo & Блок дозирования & $\begin{array}{c}\text { Система } \\
\text { управления капилляро }\end{array}$ \\
\hline $\begin{array}{c}\text { Сменный ротор } \\
\text { подачи проб }\end{array}$ & $\begin{array}{c}\text { Блок } \\
\text { позицнонировання } \\
\text { кассеты }\end{array}$ & nosmunomposannd \\
\hline 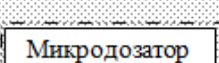 & Микродозозатор & blok \\
\hline मr & & 0орезкн капи итра \\
\hline
\end{tabular}

Fig. 2. Selection of individual units of device.

Figure 2 shows the connection of capillary control system of the immunochromatographic analysis complex with a dosing device, a block for positioning tapes with test strips, and a device for preparing and feeding samples and reagents.

\section{Capillar Control System (Capillation Positioning Unit and Cutting Unit)}

Conceptual design of the considered injection unit was based on fundamental solution of capillary control problem, namely, fixing the capillary in the automatic manipulator and moving it (the capillary positioning unit (Figure 2), movement pattern of which is shown in Figure (3) and capillary trimming (the capillary trimming unit ( Figure 2)).

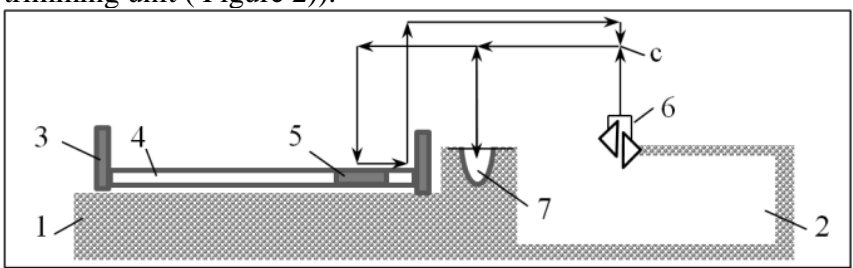

Fig.3. Capillary transfer pattern [4]

where 1 is the base, 2 is the container for collecting the waste sections of the capillary; 3 is device for precise positioning of the test cassette; 4 is test cassette with a test strip; 5 is the place on the test strip for sample application; 6 is capillary trimming unit; 7 is well dosing device; $\mathrm{c}$ is the starting point of the device operation cycle [ 15$]$.

\section{Capillary Movement Unit}

At this stage of research and formulation of concept, a technical solution implemented in 3D printers was chosen as a prototype of the principle and design of manipulator movement. This technological solution provides movement of the working head in 3 axes. The minimum sufficient at this stage of development is movement of the manipulator along two axes.

As a result, concept of the capillary positioning unit, which provides its supply, is a two-coordinate system that allows you to move the capillary along 2 axes [ Figure 3]. The movement is carried out in up-down direction to ensure the intake and discharge of liquid directly into the container, as well as movement of the capillary from sample collection capacity to the test strip and to the capillary trimming and disposal unit. The installation design assumes presence of two racks. One of which has two legs and a niche between them (about $3 \mathrm{~cm}$ in height), the dimensions of which make it possible to place an automated sample feeding device.
The use of guides and capillary movement mechanism, and presence of motors are decisive which determine dimensions of the installation. The intended use of installation as autonomous equipment also imposes requirements on its strength characteristics, and, accordingly, requirements on the material of installation elements [17] and their manufacturing technology determine certain design solutions, such as presence of stiffeners. Presence of reinforcing elements to prevent deformation and destruction of attachment points of guides are provided in this design [18]. Liquid dosing system created as a result of prototyping which uses example of a robotic injection system and detachable capillaries [19] is presented in Figure 4.

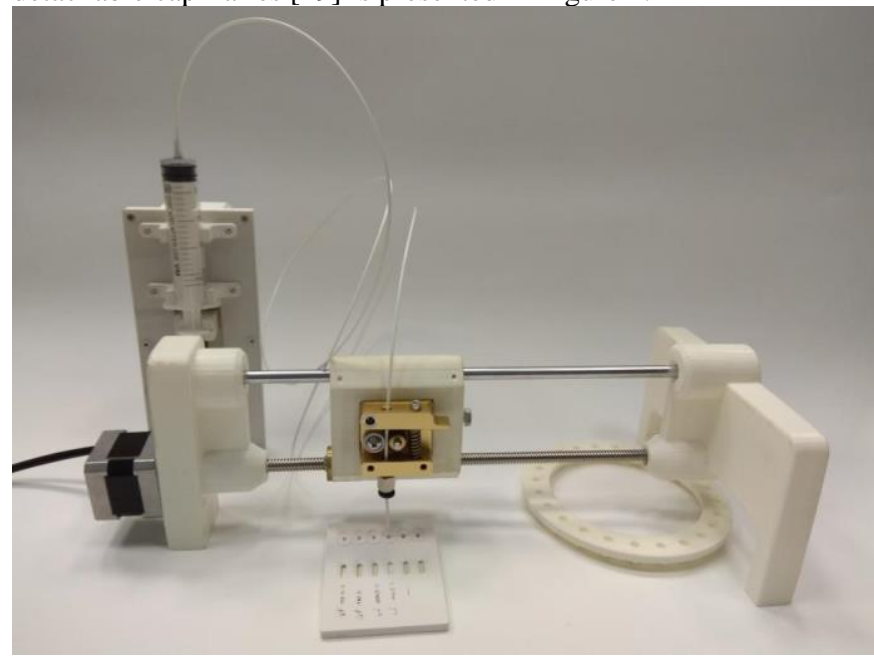

Fig. 4. Fluid dosing systems on the example of a robotic injection system using capillaries

A principle of capillary fixing on the manipulator has been developed taking into account the possibility of using a wide range of capillaries which can be different in diameter and material. Currently capillaries are available with an outer diameter of 350 to 400 microns, while the inner diameter varies from 10 to 200 microns. The capillary is clamped by pressing it with a roller to the feed gear, as can be seen in Figure 4. The capillary is controlled manually by using a spring-loaded lever.

\section{Capillar Trimming Unit}

The conceptual design of capillary trimming unit involves solving the following tasks: ensuring reliability of trimming the used end of capillary, including power spot of the cutting point, and ensuring reliable fixation of structural elements using an available reliable and well-proven consumable material.

Given that, pruning is carried out automatically and the motor ensures that about 100,000 operations are performed; it is advisable to use interchangeable cutting knives. As knives, you can use well-proven stationery knives, the blade of which is updated by breaking off the used edge of the knife. Taking into account possible efforts during the cutting operation, it is necessary to ensure reliable fastening of the knives and elements that ensure movement of knives to the base.

At this moment, a conceptual design has been developed for movement of the knife at the time of cutting capillaries (Figures 5, $6,7)$. A gear mounted on a stationary base part of the structure is fitted with a movable part with teeth on the inside of the hole. Knife is fixed on the fixed part of the structure. Movable part is attached to the base on a ball bearing with a flange, which allows you to firmly secure movable part to the base, preventing the bearing from flying. The principle of operation of this unit assumes that gear is fixed on the fixed base part, transmits movement from motor to the internal teeth of the movable part, which in turn moves the capillary pushing it onto knife blade. 
Distance from teeth of the movable part to the point of attachment refers to distance from the point of attachment to the cutting point as 1: 5. Thus, this design works on the principle of a lever, increasing force supplied by the motor, providing a reserve of power to the capillary cut-off points.

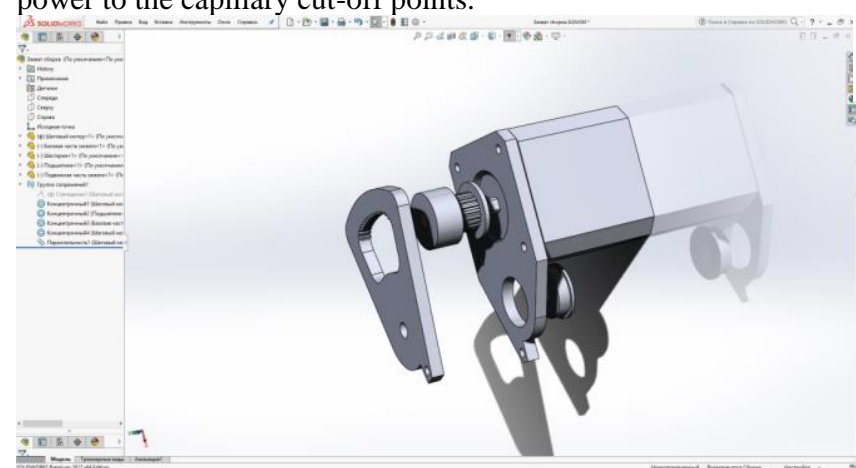

Fig.5. System to ensure movement of the knife at the time of cutting capillaries (front view)

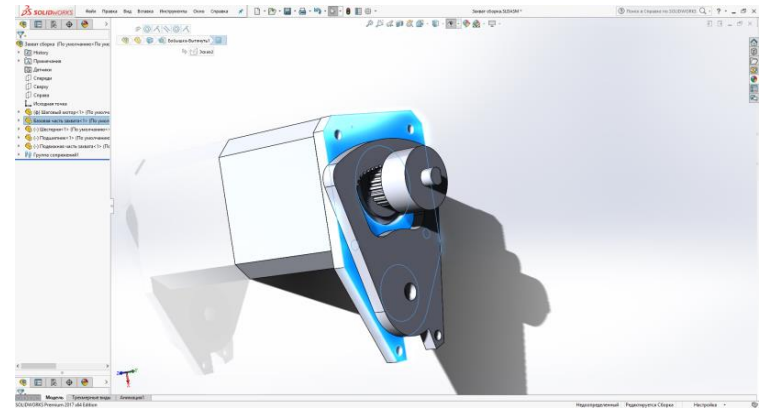

Fig. 6 System for ensuring movement of the knife at the time of cutting capillaries (complete).

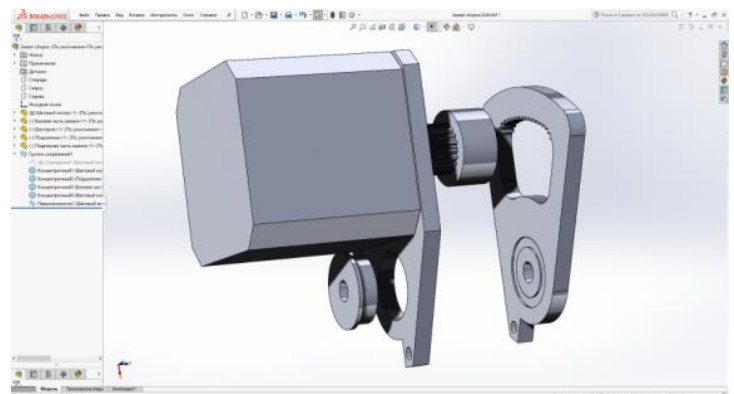

Fig. 5. System for ensuring movement of the knife at the time of cutting capillaries (rear view) - shows a ball bearing with a flange.

The cycle of using knife involves breaking edge of the knife and rearranging the new blade. Disposal of the debris from used blade of knife is supposed to be collected in the same containers as the capillary trim. The conceptual design of knife blade fixation system, which breaks off its used edge and its utilization, fixes a new edge, and allows replacing the entire knife blade, is under development.

\section{Conclusion}

Process of developing the concept can be viewed as iterative course of action, since it is necessary to revise the design of the plant as a whole each time when choosing a conceptual solution for individual units, taking into account the structural nuances of the individual units and the principles of their interaction. Thus, in the development process this approach allows one to take into account the close connection of capillary control system of immunochromatographic analysis complex with a dosing device, a block for positioning test strips cassettes, and a device for preparing and feeding samples and reagents.
As practice has shown, design development has become more complex and moved into the field of information technology [22]. At the present moment of time, it is the information system which

onıy provides new tormats or presenting ideas that are more adapted to production [ 25 ]. Due to this, rapid prototyping tools (rapid prototyping technologies) make it possible to obtain valid prototypes and test and correct design solutions in the shortest possible time.

\section{References}

[1] Patent RU 6892 U1, V. Vlasov (RU) and collaborators. A device for microdosing of liquids. The priority date is 10.06 .1997 .

[2] RU 14663 U1, Zhavner V.L. (RU). Dosing device for liquid and viscous products. Priority date 07/19/1999.

[3] RU $123516 \mathrm{U}$ 1, Chashechkin Yu.D. (RU). A device for forming one-dimensional droplets of liquid or gas bubbles. The priority date is 27.08.2012.

[4] Danilov S.A., Yezhov E.A., Moroz V.V., Gladyshev P.P. Capillary control system with no mutual contamination of samples. The 24th scientific and practical conference of students, graduate students and young professionals (with international participation). Sat materials. - Dubna, 2017. - 545-546 p.

[5] Application 081770 Russian Federation, MPK11 A G01F 11/00. Device for microdosing of dangerous biological fluids, excluding contamination of dangerous infections [Text] / PPGladyshev, V.V. Frost. - \# 2015153077: Appl. 12/10/2015; publ. 06/16/17. Byul. №17. - $1 \mathrm{~s}$.

[6] Angela M. Caliendo, David N. Gilbert, Christine C. Ginocchio, E. Kimberly, E. Hanson, Marissa, Thomas, C. Quinn, Fred C. Tenover, David Alland, J. Anne J. Better Tests, Clinical Infectious Diseases, Volume 57, Issue suppl_3, 1 December 2013, Pages S139 - S170, https: //doi.org/10.1093/cid/cit578

[7] Tille P.M. Bailey \& Scott's Diagnostic Microbiology, 14th Edition, Elsevier, St. Louies, Missouri: Elsevier.2017, 1136

[8] Frost V.V., Baulina L.V., Ibragimova S.A., Gorshkova R.M., Gladyshev P.P. Fluorescent reader for immunochromatography. Proceedings of the scientific seminar in memory of Professor, Doctor of Chemical Sciences Igor L. Khodakovsky: Sat. mater under the total ed. P.P. Gladysheva. - Dubna: State. Univ. "Dubna", 2017. - $150-155 \mathrm{p}$.

[9] Moroz V.V. Fluorescent reader for quantitative immunochromatographic analysis [Text] / V.V. Moroz, S.A. Ibragimova, V.A. Vinogradov, L.V. Baulina, R.M. Gorshkova, P.P. Gladyshev // Analytical Chromatography and Capillary electrophoresis: sat. articles. - Krasnodar, 2017. - p.24.

[10] Kazachkova, O.A., Zyabneva, O.A., Mamedova, I.Y., Kulishova, E.A. 3D technologies in the production of jewelry with elements of complicated design. International Journal of Engineering and Technology(UAE), 7 (3), pp. 155-157.

[11] Reich, Y. and Paz, A. 2008. Managing product quality, risk, and resources through resource quality function deployment. Journal of Engineering Design, 19(3): 249-267.

[12] Ruder, J. A. and Sobek, D. K. 2007. Experiment on a system-leve design tool. Journal of Engineering Design, 18(4): 327-342.

[13] Stepanova E.A., Kazachkova O.A. Design Expanding Opportunities. / International Scientific and Practical Conference: Universal Design: Different Opportunities - Comfortable Environment, November 30-December 2, Moscow, p. 121-122.

[14] Glaser, B. G. (2001). The grounded theory perspective: Conceptualization contrasted with description. San Francisco, CA: Sociology Press

[15] Baulina L.V., Sobolev I.A., Moroz V.V., Gladyshev P.P.Robotic system of immunochromatographic analysis. The 24th scientific and practical conference of students, graduate students and young professionals (with international participation). Sat materials. Dubna, 2017. - 536-537 p.

[16] Danilov S.A., Yezhov E.A., Moroz V.V., Gladyshev P.P. Disposable cassette-rotor for microcentrifugation. The 24th scientific and practical conference of students, graduate students and young professionals (with international participation). Sat materials. - Dubna, 2017. - 547 p. 
[17] Sokolova M.L., Modern Problems of the "Technology of Artistic Materials Processing" direction, Russian Technological Journal, 2017, Volume 5 No. 1, C 50-56.

[18] Thomas J.L. Van Rompay, Geke D.S. (2015) Ludden Types of Embodiment of Product Design. International Journal of Design, 9 (1), 1-11.

[19] Moroz V.V., Vinogradov V.A., Baulina L.V., Vertlina O. Microdispenser system for precise dosing of reagents. The 24th scientific and practical conference of students, graduate students and young professionals (with international participation). Sat materials. - Dubna, 2017. - 540-541 p.

[20] Buchanan, R. (2000). Good design in the digital age. AIGA Journal of Design for the Network Economy, 1 (1). 1-5

[21] Fiksel, J. R. (2009). Design for environment: A guide to sustainable product development. New York, NY: McGraw-Hill.

[22] Kushnir A.P. VISUALIZATION OF THE GEOMETRICAL PROBLEM OF CNC "VESTNIK MGTU MIREA" No. 42015 Volume II

[23] Ibragimova S.A., Dezhurov S.V., Moroz V.V., Gladyshev P.P. A new analytical platform for immunochromatographic diagnosis of diseases IDORI: physical and chemical bases for the implementation of the analytical system and new principles for processing and storing data. Proceedings of the scientific seminar in memory of Professor, Doctor of Chemical Sciences Igor L. Khodakovsky: Sat. mater / under the total ed. P.P. Gladysheva. Dubna: State. Univ. "Dubna", 2017. - 82-88 p.

[24] Lapan, S. D., \& Quartaroli, M. T. (2009). Research essentials: An introduction to design and practices. San Francisco, CA: JosseyBass.

[25] Shaofeng Liu, Iain M. Boyle 2009. Engineering design: perspectives, challenges, and recent advances. Journal of Engineering Design 20 (1), 7-19. 